\title{
HEMATOLOGICAL AND BIOCHEMICAL EFFECTS OF PARTIAL AND COMPLETE SPLENECTOMY IN DOGS
}

\author{
IBRAHIM I. ELSHAHAWY ${ }^{1}$, KORITTUM, A.S. ${ }^{2}$ and LEBDA, M. ${ }^{3}$ \\ ${ }^{1}$ Department of Animal Medicine, Faculty of Veterinary Medicine, Alexandria University, Egypt. \\ ${ }^{2}$ Department of Surgery, Faculty of Veterinary Medicine, Alexandria University, Egypt. \\ ${ }^{3}$ Department of Biochemistry Faculty of Veterinary Medicine, Alexandria University, Egypt.
}

Received: 28 March 2018; Accepted: 17 April 2018

\begin{abstract}
The purpose of the current study was to describe the effect of surgical splenectomy technique either partial or complete on some biochemical and hematological parameters. Six male mature mongrel dogs were subdivided into two groups (complete and partial splenectomy), and six immature mongrel dogs were subdivided into two groups (complete and partial splenectomy). Blood samples were taken before (base line), 10, 30, and 60 days post-operative. The results showed a significant reduction in serum albumin and iron levels 10 days after partial and complete splenectomy in both immature and mature dogs. However, serum gamma globulin fraction, ferritin, transferrin, and ceruloplasmin concentrations were significantly increased at the same period following surgical operation. There was a perturbation in serum activity of transaminases, bilirubin, and urea levels. The levels of WBCs, neutrophils, lymphocytes, $\mathrm{MCH}, \mathrm{MCHC}$, and platelets in completely splenectomized immature and mature dogs were increased. While, the RBCs and $\mathrm{Hb}$ concentrations were significantly decreased following complete splenectomy in immature and mature dogs. These results concluded that splenectomy in dogs may induce anemia, thrombosis, and inflammatory conditions but also increase cellular and humeral immunity as a compensatory mechanism.
\end{abstract}

Key words: Splenectomy, transferrin, platelets, ceruloplasmin, dogs

\section{INTRODUCTION}

The spleen, one of the most vascularized organs of the body and consider as a part of the mononuclear phagocyte system which is located between the portal and systemic circulations. Its blood supply comes from the splenic artery and from a rich arterial collateral network, mainly splenogastric, while its venous drainage flows to the splenic vein and a small part to the splenogastric veins. The splenic vein joins the superior mesenteric vein to form the portal vein (Petroianu, 2003). The spleen plays important functions, including hematopoiesis, cell purification and reservoir of blood elements (Sipka et al., 2006).

The available information concerning the results of splenectomy in the veterinary field is not wellidentified and most of the knowledge in this consideration has originated from human provenance (Chaudhry et al., 1997). The indications for total splenectomy include tumors, benign splenic lesions,

Corresponding author: Dr. LEBDA, M.

E-mail address: biochemistry232@yahoo.com

Present address: Department of Biochemistry Faculty of Veterinary Medicine, Alexandria University, Egypt. splenic torsion, traumatism and hematological immune-mediated diseases not responsive to treatments with drugs (Harari, 1999). Hemangiosarcoma (HSA) is the most common tumor of the canine spleen (Tillson, 2003). Partial splenectomy is indicated in animals with traumatic or focal lesions of the spleen to preserve splenic function. Complete removal of the spleen may lead to undesirable effect such as post-operative infections and sepsis, due to the reduce production of antibodies and phagocytes or may lead to thrombosis, due to rose of platelet count in blood (Khan et al., 2009).

Despite the extensive literature on the effects splenectomy in human with different illness such as thalassemia, liver injuries, HCV infection and others on hematological and biochemical parameters, there is a few data about the hematological and biochemical alterations following splenectomy in animals specifically dogs. The aim of this study is to investigate the effects of total and partial splenectomy in apparently healthy adult and young dogs on complete blood count, liver functions (ALT, AST, albumin, and bilirubin), urea, and iron balance (iron, ferritin, ceruloplasmin, and transferrin) levels. 


\section{MATERIALS AND METHODS}

\section{Animals and experimental design}

Twelve apparently healthy female stray dogs, Six puppies aging from (3-4 month), weighting (4-6 kg) and others aging from (3-4 years) and weighting $(20-25 \mathrm{~kg})$ were used in the present study which approved by Institutional Animal Use and Care Committee of Faculty of Veterinary Medicine, Alexandria University. The local ethics committee for the use of laboratory animals approved all experimental procedures. Before experiment the animals were clinically and ultrasonoghraphically examined to ensure that they are apparently healthy. Animals were randomly divided into four groups according to age 2 mature groups and 2 immature groups ( $\mathrm{n}=3$ in each): group $\mathbf{A}$, mature with complete splenectomy, group $\mathbf{B}$, mature with partial splenectomy, group C, immature with complete splenectomy, group D, immature with partial splenectomy. The animal experiments were carried out in accordance with the National Institutes of Health (NIH) Guidelines for the Care and Use of Laboratory Animals, and the study protocol was approved by ethics committee, Faculty of Veterinary Medicine, Alexandria University.

\section{Surgical operation}

Food was withheld from all the dogs $12 \mathrm{~h}$ and water $2 \mathrm{~h}$ before the experiment. The dogs were premedicated pre-operatively by intramuscular administration of xylazine $\mathrm{HCl}$ as sedation $1 \mathrm{mg} / \mathrm{kg}$ (Xyla-ject $\circledast$, Adwia Pharmaceuticals Co., Egypt). Fifteen minutes after pre-medication, induction of general anesthesia was induced and maintained with $5 \mathrm{mg} / \mathrm{kg}$ b.wt Ketamine $\mathrm{HCl}$ (Ketamine ${ }^{\circledR} 10 \%$, Alfasan Pharmaceuticals, Holland) injected intramuscular.

The ventral abdomen prepared for aseptic surgery, a ventral midline abdominal incision from the xiphoid, extending caudal to the umbilicus were made in premature dogs, Otherwise in adult animals left subcostal approach were made. After exposure of the spleen and isolated extra abdominal and place moistened abdominal laparotomy pads around the incision under the spleen, for total splenectomy proximal vessels of the hilum were isolated. Double ligate and transect all vessels at the splenic hilus with absorbable suture material polyglactin 910 (Vicryl®).

\section{Blood samples}

Two blood samples of each dog were taken from the cephalic vein just before surgery and on the $10^{\text {th }}$ day, 1 month and 2 month postoperatively. First sample was collected into the Voma Med® tubes containing $\mathrm{K}_{3}$ EDTA anticoagulant to obtaining whole blood for hematological examination including red blood cell count (RBC), white blood cell count (WBC), hemoglobin concentration $(\mathrm{Hb})$, mean corpuscular volume (MCV), mean corpuscular hemoglobin $(\mathrm{MCH})$, mean corpuscular hemoglobin concentration (MCHC), platelet count (PLT), neutrophils and lymphocyte counts were determined using automated blood analyzer (SERONO-9120 Baker System). Second blood sample was taken in plain Voma Med® tubes without anticoagulant for obtaining serum for measurement of ALT, AST, bilirubin, urea, iron, ferritin, ceruloplasmin, transferrin, and serum protein electrophoresis.

\section{Biochemical analysis}

Serum liver marker enzymes (ALT, AST), bilirubin, and urea concentration determined using Biodiagnostic kits according to the manufacturer's instructions. Serum ceruloplasmin forms a precipitate with a specific antiserum which was determined using immunoturbidimetric assay (Wolf, 1982). Serum transferrin was immunoturbidimetric determined (Lizana and Hellsing, 1974). Ferritin level was estimated according to (Finch et al., 1986). Electrophoretic pattern of serum protein was evaluated according to (Kaplan and Savory, 1965).

\section{Statistical analysis}

Data were analyzed using the SPSS package (SPSS Inc., Chicago, IL). Results are expressed as mean \pm SE with the experiment repeated at least three times. Statistical evaluations were done using one-way analysis of variance (ANOVA). A $p$ value of $<0.05$ was considered significant.

\section{RESULTS}

\section{Surgical operation}

The ventral midline celiotomy was standard access for splenectomy in premature dogs; the spleen was easily and directly located. However a left paracostal approach in mature dogs was found to be superior to midline approaches for removal of the spleen. The paracostal abdominal approach provides excellent exposure, ease for manipulation, reduced anesthetic time, and fewer related problems such as postoperative pain with minimal need for analgesic and rapid return to normal function.

\section{Serum protein fractionation}

There was a significant reduction of serum albumin level 10 days after partial and complete splenectomy in young and adult dogs $(P<0.05)$, which tend to be increased to reach normal level at 60 days in young dogs while still below normal level in adult dogs. The change in albumin concentration was corresponding to the difference in serum total protein level (table 1). On the other hand, serum gamma globulin level tends to be increased 30, 60 days after partial and complete splenectomy in young and adult dogs at $P<0.05$ (table 1). 


\section{Iron, ferritin, transferrin, and ceruloplasmin concentrations}

The level of serum iron was significantly decreased following partial or complete splenectomy and gradually increased to reach its level at 0 day in both young and adult dogs $(P<0.05)$. However, serum ferritin, transferrin, and ceruloplasmin concentrations were significantly increased 10 days after both operation and tend to be decreased to reach the normal levels at 60 day (table 2).

\section{Liver and kidneys functions}

The data represented in table (3) showed that serum activities of ALT, AST were significantly increased following partial or complete splenectomy without change in serum bilirubin level. However, serum urea concentration was significantly increased 30, and 60 days post-operative technique in young and adult dogs.

\section{Hematological parameters}

The levels of WBCs, neutrophils, lymphocytes, and platelets in completely splenectomized young and adult dogs were significantly increased soon after operation followed by gradual decrease toward the normal values at the end of experiment (table 5). While in partially splenectomized small and large dogs, their levels significantly increase after operation and reach to normal levels at two month.

The MCV, and MCHC in all groups significantly increase after operation followed by gradual decrease toward normal, except in partially splenectomized adult dogs have no significant changes. While $\mathrm{MCH}$ in completely splenectomized young and large dogs showed significant increase along the experiment period, while, partially splenectomized dogs showed non-significant changes.

$\mathrm{RBCs}$ and $\mathrm{Hb}$ significantly decrease in completely splenectomized small and large dogs and start increasing toward normal levels at the end of experiment. While in partially splenectomized dogs, they slightly decrease after experiment and rapidly returned to normal values (table 4).

Table 1: Serum protein fractionation after partial and complete splenectomy in young and adult dogs

\begin{tabular}{|c|c|c|c|c|c|c|c|c|}
\hline & & $\begin{array}{c}\text { Period of } \\
\text { operation } \\
\text { (days) }\end{array}$ & $\begin{array}{l}\text { T. protein } \\
\text { (g/dl) }\end{array}$ & $\begin{array}{l}\text { Albumin } \\
(\mathrm{g} / \mathrm{dl})\end{array}$ & $\underset{(\mathrm{g} / \mathrm{dl})}{\text { Alpha1 }}$ & $\underset{(\mathrm{g} / \mathrm{dl})}{\text { Alpha2 }}$ & $\begin{array}{l}\text { Beta } \\
\text { (g/dl) }\end{array}$ & $\begin{array}{c}\text { Gamma } \\
(\mathrm{g} / \mathrm{dl})\end{array}$ \\
\hline \multirow{8}{*}{$\begin{array}{l}\text { Young } \\
\text { dogs }\end{array}$} & \multirow{4}{*}{$\begin{array}{l}\text { Complete } \\
\text { splenoctomy }\end{array}$} & 0 & $5.37 \pm 0.15^{\mathbf{b}}$ & $3.35 \pm 0.05^{\mathbf{a}}$ & $0.18 \pm 0.00^{\mathbf{c}}$ & $0.87 \pm 0.06^{\mathbf{b}}$ & $0.76 \pm 0.04^{\mathrm{a}}$ & $0.22 \pm 0.01^{\mathbf{b}}$ \\
\hline & & 10 & $5.30 \pm 0.12^{\mathbf{b}}$ & $3.32 \pm 0.05^{\mathrm{a}}$ & $0.21 \pm 0.00^{\mathbf{b}}$ & $0.83 \pm 0.04^{\mathbf{b}}$ & $0.71 \pm 0.04^{\mathrm{a}}$ & $0.22 \pm 0.00^{\mathbf{b}}$ \\
\hline & & 30 & $5.20 \pm 0.17^{\mathbf{b}}$ & $3.00 \pm 0.06^{\mathbf{b}}$ & $0.17 \pm 0.00^{\mathbf{c}}$ & $1.08 \pm 0.05^{\mathrm{a}}$ & $0.72 \pm 0.06^{\mathbf{a}}$ & $0.23 \pm 0.00^{\mathbf{b}}$ \\
\hline & & 60 & $6.00 \pm 0.17^{\mathrm{a}}$ & $3.47 \pm 0.06^{\mathbf{a}}$ & $0.23 \pm 0.01^{\mathrm{a}}$ & $1.23 \pm 0.06^{\mathrm{a}}$ & $0.81 \pm 0.05^{\mathrm{a}}$ & $0.26 \pm 0.01^{\mathrm{a}}$ \\
\hline & \multirow{4}{*}{$\begin{array}{l}\text { Partial } \\
\text { splenoctomy }\end{array}$} & 0 & $6.20 \pm 0.12^{\mathrm{a}}$ & $3.72 \pm 0.06^{\mathbf{a}}$ & $0.28 \pm 0.00^{\mathrm{a}}$ & $1.00 \pm 0.01^{\mathrm{a}}$ & $0.85 \pm 0.05^{\mathrm{a}}$ & $0.35 \pm 0.01^{\mathbf{b}}$ \\
\hline & & 10 & $5.60 \pm 0.23^{b c}$ & $3.21 \pm 0.06^{\mathbf{b}}$ & $0.19 \pm 0.00^{\mathbf{c}}$ & $0.98 \pm 0.06^{\mathbf{a}}$ & $0.83 \pm 0.05^{\mathrm{a}}$ & $0.36 \pm 0.04^{\mathbf{b}}$ \\
\hline & & 30 & $5.50 \pm 0.12^{c}$ & $3.17 \pm 0.05^{\mathbf{b}}$ & $0.24 \pm 0.00^{\mathbf{b}}$ & $0.83 \pm 0.03^{\mathbf{b}}$ & $0.79 \pm 0.01^{\mathrm{a}}$ & $0.44 \pm 0.01^{\mathrm{a}}$ \\
\hline & & 60 & $6.10 \pm 0.17^{\mathrm{ab}}$ & $3.79 \pm 0.07^{\mathrm{a}}$ & $0.23 \pm 0.01^{\mathbf{b}}$ & $0.93 \pm 0.05^{\mathrm{ab}}$ & $0.84 \pm 0.06^{\mathrm{a}}$ & $0.30 \pm 0.01^{\mathbf{b}}$ \\
\hline \multirow{8}{*}{$\begin{array}{c}\text { Adult } \\
\text { dogs }\end{array}$} & \multirow{4}{*}{$\begin{array}{l}\text { Complete } \\
\text { splenoctomy }\end{array}$} & 0 & $7.20 \pm 0.12^{\mathrm{a}}$ & $4.15 \pm 0.04^{\mathrm{a}}$ & $0.26 \pm 0.00^{\mathrm{a}}$ & $1.26 \pm 0.05^{\mathrm{ab}}$ & $1.22 \pm 0.00^{\mathrm{a}}$ & $0.28 \pm 0.01^{\mathrm{d}}$ \\
\hline & & 10 & $6.80 \pm 0.17^{\mathbf{b}}$ & $3.52 \pm 0.06^{\mathbf{b}}$ & $0.26 \pm 0.01^{\mathrm{a}}$ & $1.38 \pm 0.06^{\mathrm{a}}$ & $1.37 \pm 0.09^{\mathrm{a}}$ & $0.34 \pm 0.01^{\mathrm{c}}$ \\
\hline & & 30 & $5.80 \pm 0.12^{c}$ & $2.78 \pm 0.05^{\mathrm{d}}$ & $0.23 \pm 0.00^{\mathbf{b}}$ & $1.06 \pm 0.01^{\mathrm{c}}$ & $1.22 \pm 0.06^{\mathrm{a}}$ & $0.51 \pm 0.01^{\mathrm{a}}$ \\
\hline & & 60 & $6.50 \pm 0.06^{\mathbf{b}}$ & $3.26 \pm 0.05^{\mathbf{c}}$ & $0.22 \pm 0.00^{\mathbf{b}}$ & $1.18 \pm 0.01^{\mathrm{bc}}$ & $1.41 \pm 0.01^{\mathrm{a}}$ & $0.42 \pm 0.01^{\mathbf{b}}$ \\
\hline & \multirow{4}{*}{$\begin{array}{c}\text { Partial } \\
\text { splenoctomy }\end{array}$} & 0 & $5.90 \pm 0.06^{\mathbf{a b}}$ & $3.28 \pm 0.05^{\mathrm{a}}$ & $0.15 \pm 0.00^{\mathbf{c}}$ & $0.93 \pm 0.01^{\mathbf{b}}$ & $1.01 \pm 0.00^{\mathbf{b}}$ & $0.54 \pm 0.00^{d}$ \\
\hline & & 10 & $6.10 \pm 0.12^{\mathrm{a}}$ & $2.69 \pm 0.06^{\mathbf{b}}$ & $0.30 \pm 0.01^{\mathrm{a}}$ & $1.12 \pm 0.00^{\mathrm{a}}$ & $1.26 \pm 0.06^{\mathrm{a}}$ & $0.74 \pm 0.00^{\mathrm{a}}$ \\
\hline & & 30 & $5.60 \pm 0.17^{\mathbf{b}}$ & $2.32 \pm 0.06^{\mathbf{c}}$ & $0.27 \pm 0.01^{\mathbf{b}}$ & $1.14 \pm 0.06^{\mathrm{a}}$ & $1.16 \pm 0.05^{\mathrm{a}}$ & $0.71 \pm 0.00^{\mathbf{b}}$ \\
\hline & & 60 & $5.50 \pm 0.12^{\mathbf{b}}$ & $2.82 \pm 0.05^{\mathbf{b}}$ & $0.14 \pm 0.00^{\mathbf{c}}$ & $0.96 \pm 0.06^{\mathbf{b}}$ & $0.97 \pm 0.01^{\mathbf{b}}$ & $0.62 \pm 0.01^{\mathrm{c}}$ \\
\hline
\end{tabular}

Values are expressed as mean \pm SE.

The values with different superscript letters within the same column significantly differ at $p<0.05$. 
Table 2: Serum iron, ferritin, transferrin, and ceruloplasmin levels after partial and complete splenectomy in young and adult dogs.

\begin{tabular}{|c|c|c|c|c|c|c|}
\hline & & $\begin{array}{c}\text { Period of } \\
\text { operation } \\
\text { (days) }\end{array}$ & $\begin{array}{c}\text { Iron } \\
(\mu \mathrm{g} / \mathrm{dl})\end{array}$ & $\begin{array}{l}\text { Ferritin } \\
(\mathrm{ng} / \mathrm{ml})\end{array}$ & $\begin{array}{l}\text { Ceruloplasmin } \\
\qquad(\mathrm{ng} / \mathrm{ml})\end{array}$ & $\begin{array}{c}\text { Transferrin } \\
(\mathbf{n g} / \mathrm{ml})\end{array}$ \\
\hline \multirow{8}{*}{$\begin{array}{l}\text { Young } \\
\text { dogs }\end{array}$} & \multirow{4}{*}{$\begin{array}{c}\text { Complete } \\
\text { splenoctomy }\end{array}$} & 0 & $163.67 \pm 4.26^{\mathrm{a}}$ & $97.17 \pm 6.81^{\mathrm{c}}$ & $19.10 \pm 1.22^{\mathrm{c}}$ & $182.33 \pm 1.79^{d}$ \\
\hline & & 10 & $107.80 \pm 2.25^{\mathbf{b}}$ & $117.67 \pm 3.52^{b}$ & $29.40 \pm 1.21^{\mathrm{a}}$ & $219.47 \pm 1.08^{b}$ \\
\hline & & 30 & $62.97 \pm 4.49^{\mathrm{d}}$ & $145.30 \pm 2.59^{\mathrm{a}}$ & $23.77 \pm 0.75^{b}$ & $206.07 \pm 2.22^{\mathrm{a}}$ \\
\hline & & 60 & $75.90 \pm 3.23^{\mathrm{c}}$ & $158.53 \pm 3.95^{\mathrm{a}}$ & $19.33 \pm 0.43^{\mathrm{c}}$ & $195.67 \pm 2.34^{\mathrm{c}}$ \\
\hline & \multirow{4}{*}{$\begin{array}{c}\text { Partial } \\
\text { splenoctomy }\end{array}$} & 0 & $171.27 \pm 3.03^{\mathbf{b}}$ & $178.97 \pm 3.61^{\mathrm{a}}$ & $17.80 \pm 1.44^{\mathrm{c}}$ & $197.73 \pm 2.69^{b}$ \\
\hline & & 10 & $89.80 \pm 2.21^{\mathrm{c}}$ & $187.90 \pm 4.31^{\mathrm{a}}$ & $35.47 \pm 1.65^{\mathrm{a}}$ & $229.23 \pm 3.26^{\mathbf{a}}$ \\
\hline & & 30 & $134.90 \pm 6.33^{\mathrm{a}}$ & $166.90 \pm 3.10^{\mathbf{b}}$ & $27.60 \pm 1.12^{b}$ & $208.20 \pm 1.97^{b}$ \\
\hline & & 60 & $170.43 \pm 2.05^{\mathrm{a}}$ & $95.67 \pm 3.32^{c}$ & $19.80 \pm 0.89^{c}$ & $198.50 \pm 4.50^{b}$ \\
\hline \multirow{8}{*}{$\begin{array}{l}\text { Adult } \\
\text { dogs }\end{array}$} & \multirow{4}{*}{$\begin{array}{c}\text { Complete } \\
\text { splenoctomy }\end{array}$} & 0 & $169.37 \pm 1.41^{\mathrm{a}}$ & $112.33 \pm 5.41^{\mathrm{c}}$ & $21.03 \pm 0.74^{\mathrm{c}}$ & $198.13 \pm 1.33^{b}$ \\
\hline & & 10 & $94.77 \pm 1.02^{\mathrm{d}}$ & $228.53 \pm 9.79^{\mathrm{a}}$ & $37.20 \pm 1.45^{\mathrm{a}}$ & $201.60 \pm 1.13^{b}$ \\
\hline & & 30 & $107.27 \pm 1.19^{c}$ & $137.73 \pm 3.49^{\mathbf{b}}$ & $28.43 \pm 0.55^{\mathbf{b}}$ & $215.60 \pm 1.10^{\mathrm{a}}$ \\
\hline & & 60 & $124.03 \pm 1.83^{\mathbf{b}}$ & $139.13 \pm 5.09^{\mathbf{b}}$ & $22.07 \pm 0.90^{\mathrm{c}}$ & $199.87 \pm 2.30^{b}$ \\
\hline & \multirow{4}{*}{$\begin{array}{c}\text { Partial } \\
\text { splenoctomy }\end{array}$} & 0 & $132.67 \pm 2.89^{\mathrm{a}}$ & $142.37 \pm 3.15^{\mathrm{c}}$ & $20.10 \pm 1.23^{c}$ & $188.73 \pm 3.39^{d}$ \\
\hline & & 10 & $90.83 \pm 1.84^{\mathrm{d}}$ & $186.10 \pm 6.12^{\mathrm{a}}$ & $33.47 \pm 1.04^{\mathrm{a}}$ & $234.17 \pm 2.43^{\mathrm{a}}$ \\
\hline & & 30 & $105.77 \pm 2.49^{\mathbf{c}}$ & $163.20 \pm 4.40^{\mathbf{b}}$ & $23.60 \pm 1.10^{b}$ & $219.03 \pm 1.47^{b}$ \\
\hline & & 60 & $120.17 \pm 1.82^{\mathbf{b}}$ & $168.80 \pm 3.70^{\mathbf{b}}$ & $20.50 \pm 0.61 b^{c}$ & $209.13 \pm 1.46^{\mathbf{c}}$ \\
\hline
\end{tabular}

Values are expressed as mean \pm SE.

The values with different superscript letters within the same column significantly differ at $p<0.05$.

Table 3: Liver and kidney functions after splenectomy after partial and complete splenectomy in young and adult dogs

\begin{tabular}{|c|c|c|c|c|c|c|}
\hline & & $\begin{array}{c}\text { Period of } \\
\text { operation } \\
\text { (days) }\end{array}$ & $\begin{array}{l}\mathbf{A L T} \\
(\mathbf{U} / \mathbf{L})\end{array}$ & $\begin{array}{l}\text { AST } \\
(\mathbf{U} / \mathbf{L})\end{array}$ & $\begin{array}{l}\text { Bilirubin } \\
\text { (mg/dl) }\end{array}$ & Urea (mg/dl) \\
\hline \multirow{8}{*}{$\begin{array}{c}\text { Young } \\
\text { dogs }\end{array}$} & \multirow{4}{*}{$\begin{array}{c}\text { Complete } \\
\text { splenoctomy }\end{array}$} & 0 & $20.43 \pm 0.69^{\mathrm{a}}$ & $14.73 \pm 0.55^{\mathbf{b}}$ & $0.90 \pm 0.03^{\mathrm{a}}$ & $17.23 \pm 1.78^{\mathrm{c}}$ \\
\hline & & 10 & $11.23 \pm 0.48^{\mathbf{c}}$ & $20.70 \pm 0.38^{\mathbf{a}}$ & $0.71 \pm 0.01^{\mathbf{b}}$ & $26.43 \pm 1.62^{b}$ \\
\hline & & 30 & $16.43 \pm 1.21^{\mathrm{b}}$ & $21.33 \pm 0.58^{\mathrm{a}}$ & $0.56 \pm 0.03^{\mathrm{c}}$ & $35.00 \pm 1.99^{\mathrm{a}}$ \\
\hline & & 60 & $11.50 \pm 1.65^{\mathrm{c}}$ & $20.60 \pm 0.69^{\mathrm{a}}$ & $0.74 \pm 0.03^{\mathbf{b}}$ & $13.70 \pm 0.59^{c}$ \\
\hline & \multirow{4}{*}{$\begin{array}{c}\text { Partial } \\
\text { splenoctomy }\end{array}$} & 0 & $8.13 \pm 1.00^{c}$ & $8.70 \pm 0.46^{\mathbf{b}}$ & $0.91 \pm 0.04^{\mathrm{c}}$ & $17.67 \pm 1.79^{c}$ \\
\hline & & 10 & $26.27 \pm 2.22^{\mathrm{a}}$ & $11.20 \pm 0.40^{\mathrm{a}}$ & $0.56 \pm 0.03^{b}$ & $17.90 \pm 2.20^{\mathrm{c}}$ \\
\hline & & 30 & $12.93 \pm 1.65^{\mathbf{b c}}$ & $11.53 \pm 0.38^{\mathrm{a}}$ & $0.74 \pm 0.03^{\mathrm{a}}$ & $26.47 \pm 2.48^{\mathbf{b}}$ \\
\hline & & 60 & $17.57 \pm 1.07^{\mathbf{b}}$ & $11.73 \pm 0.38^{\mathrm{a}}$ & $0.85 \pm 0.03^{\mathrm{a}}$ & $44.27 \pm 3.32^{\mathrm{a}}$ \\
\hline \multirow{8}{*}{$\begin{array}{l}\text { Adult } \\
\text { dogs }\end{array}$} & \multirow{4}{*}{$\begin{array}{c}\text { Complete } \\
\text { splenoctomy }\end{array}$} & 0 & $15.13 \pm 0.38^{\mathrm{a}}$ & $15.30 \pm 0.53^{\mathbf{a b}}$ & $0.88 \pm 0.02^{\mathrm{a}}$ & $15.67 \pm 1.04^{\mathbf{b}}$ \\
\hline & & 10 & $5.80 \pm 0.46^{\mathbf{b}}$ & $17.70 \pm 1.27^{\mathrm{a}}$ & $0.68 \pm 0.02^{b}$ & $15.07 \pm 1.37^{\mathbf{b}}$ \\
\hline & & 30 & $3.53 \pm 0.22^{\mathrm{c}}$ & $10.77 \pm 0.41^{\mathrm{c}}$ & $0.68 \pm 0.05^{b}$ & $22.90 \pm 2.47^{\mathrm{a}}$ \\
\hline & & 60 & $5.53 \pm 0.28^{\mathbf{b}}$ & $13.60 \pm 0.64^{b}$ & $0.82 \pm 0.02^{\mathrm{a}}$ & $24.67 \pm 2.03^{\mathrm{a}}$ \\
\hline & \multirow{4}{*}{$\begin{array}{c}\text { Partial } \\
\text { splenoctomy }\end{array}$} & 0 & $11.27 \pm 1.24^{\mathrm{a}}$ & $18.40 \pm 1.04^{b}$ & $0.93 \pm 0.04^{\mathrm{a}}$ & $20.47 \pm 1.79^{a}$ \\
\hline & & 10 & $7.97 \pm 0.78^{\mathbf{b c}}$ & $22.40 \pm 0.84^{\mathrm{a}}$ & $0.68 \pm 0.05^{\mathbf{c}}$ & $19.03 \pm 1.56^{\mathrm{a}}$ \\
\hline & & 30 & $10.10 \pm 0.46^{\mathbf{a b}}$ & $13.73 \pm 0.41^{\mathrm{c}}$ & $0.77 \pm 0.02^{\text {bc }}$ & $20.53 \pm 0.67^{\mathrm{a}}$ \\
\hline & & 60 & $5.73 \pm 0.09^{c}$ & $12.57 \pm 1.24^{\mathbf{c}}$ & $0.88 \pm 0.02^{\mathbf{a b}}$ & $14.23 \pm 0.96^{\mathbf{b}}$ \\
\hline
\end{tabular}

Values are expressed as mean \pm SE.

The values with different superscript letters within the same column significantly differ at $p<0.05$. 
Table 4: Hemoglobin and blood indices after after partial and complete splenectomy in young and adult dogs

\begin{tabular}{|c|c|c|c|c|c|c|c|}
\hline & & $\begin{array}{c}\text { Period of } \\
\text { operation } \\
\text { (days) }\end{array}$ & $\begin{array}{c}\text { RBCs } \\
\left(10^{6} / \mu l\right)\end{array}$ & $\begin{array}{l}\text { Hb } \\
(\mathrm{g} / \mathrm{dl})\end{array}$ & $\begin{array}{c}\text { MCV } \\
\text { (fl) }\end{array}$ & $\begin{array}{c}\text { MCH } \\
(\mathbf{p g})\end{array}$ & $\begin{array}{c}\text { МCHC } \\
(\%)\end{array}$ \\
\hline \multirow{8}{*}{$\begin{array}{l}\text { Young } \\
\text { dogs }\end{array}$} & \multirow{4}{*}{$\begin{array}{c}\text { Complete } \\
\text { splenoctomy }\end{array}$} & 0 & $5.49 \pm 0.17^{\mathbf{a}}$ & $12.10 \pm 0.15^{\mathrm{a}}$ & $62.37 \pm 0.52^{b}$ & $21.03 \pm 0.38^{\mathbf{c}}$ & $34.23 \pm 0.52^{\mathrm{ab}}$ \\
\hline & & 10 & $4.28 \pm 0.18^{b}$ & $11.07 \pm 0.24^{\mathrm{ab}}$ & $64.17 \pm 0.59^{\mathbf{a b}}$ & $22.93 \pm 0.13^{b}$ & $35.57 \pm 0.44^{\mathrm{a}}$ \\
\hline & & 30 & $3.98 \pm 0.02^{b}$ & $10.33 \pm 0.42^{\mathbf{b}}$ & $65.07 \pm 0.58^{\mathrm{a}}$ & $23.67 \pm 0.15^{b}$ & $31.90 \pm 1.04^{\mathbf{b}}$ \\
\hline & & 60 & $4.06 \pm 0.08^{b}$ & $10.77 \pm 0.38^{\mathbf{b}}$ & $62.37 \pm 0.44^{\mathbf{b}}$ & $25.70 \pm 0.21^{\mathrm{a}}$ & $33.43 \pm 0.62^{a b}$ \\
\hline & \multirow{4}{*}{$\begin{array}{c}\text { Partial } \\
\text { splenoctomy }\end{array}$} & 0 & $4.90 \pm 0.12^{\mathrm{a}}$ & $9.77 \pm 0.20^{\mathrm{a}}$ & $65.67 \pm 0.76^{\mathbf{b}}$ & $21.50 \pm 0.87^{\mathbf{a}}$ & $31.13 \pm 0.55^{c}$ \\
\hline & & 10 & $4.47 \pm 0.22^{\mathrm{ab}}$ & $9.27 \pm 0.19^{\mathbf{a b}}$ & $72.03 \pm 0.46^{\mathrm{a}}$ & $22.27 \pm 0.61^{\mathrm{a}}$ & $32.83 \pm 0.68^{\mathbf{b}}$ \\
\hline & & 30 & $4.20 \pm 0.15^{\mathrm{ab}}$ & $9.00 \pm 0.12^{b}$ & $61.67 \pm 0.95^{c}$ & $22.83 \pm 0.49^{\mathrm{a}}$ & $35.97 \pm 0.26^{\mathbf{a}}$ \\
\hline & & 60 & $4.50 \pm 0.15^{b}$ & $9.73 \pm 0.12^{\mathrm{a}}$ & $65.90 \pm 0.17^{\mathbf{b}}$ & $22.53 \pm 0.35^{\mathrm{a}}$ & $33.60 \pm 0.29^{b}$ \\
\hline \multirow{8}{*}{$\begin{array}{l}\text { Adult } \\
\text { dogs }\end{array}$} & \multirow{4}{*}{$\begin{array}{c}\text { Complete } \\
\text { splenoctomy }\end{array}$} & 0 & $7.17 \pm 0.18^{\mathbf{a}}$ & $12.33 \pm 0.32^{\mathrm{a}}$ & $62.50 \pm 0.35^{\mathrm{c}}$ & $18.57 \pm 0.58^{\mathbf{b}}$ & $29.33 \pm 0.41^{\mathbf{b}}$ \\
\hline & & 10 & $5.33 \pm 0.15^{\mathrm{c}}$ & $10.17 \pm 0.18^{\mathbf{b}}$ & $60.23 \pm 0.24^{d}$ & $20.73 \pm 0.79^{a}$ & $30.77 \pm 0.49^{\mathbf{a b}}$ \\
\hline & & 30 & $5.83 \pm 0.15^{\text {bc }}$ & $11.63 \pm 0.38^{\mathrm{a}}$ & $67.20 \pm 0.62^{a}$ & $21.80 \pm 0.56^{\mathbf{a}}$ & $31.57 \pm 0.44^{\mathrm{a}}$ \\
\hline & & 60 & $5.97 \pm 0.18^{b}$ & $12.00 \pm 0.26^{\mathrm{a}}$ & $64.17 \pm 0.18^{b}$ & $21.60 \pm 0.45^{\mathrm{a}}$ & $30.43 \pm 0.46^{\mathrm{ab}}$ \\
\hline & \multirow{4}{*}{$\begin{array}{c}\text { Partial } \\
\text { splenoctomy }\end{array}$} & 0 & $6.90 \pm 0.25^{\mathrm{a}}$ & $13.23 \pm 0.39^{\mathrm{a}}$ & $72.67 \pm 0.88^{\mathrm{a}}$ & $23.13 \pm 0.99^{\mathrm{a}}$ & $31.83 \pm 0.79^{a}$ \\
\hline & & 10 & $5.53 \pm 0.22^{b}$ & $11.50 \pm 0.23^{\mathbf{b}}$ & $70.53 \pm 0.47^{\mathbf{b}}$ & $24.73 \pm 1.34^{\mathrm{a}}$ & $31.53 \pm 0.64^{\mathrm{a}}$ \\
\hline & & 30 & $6.23 \pm 0.15^{\mathrm{a}}$ & $12.23 \pm 0.41^{\mathbf{a b}}$ & $73.53 \pm 0.35^{\mathrm{a}}$ & $22.77 \pm 0.93^{\mathrm{a}}$ & $32.13 \pm 0.23^{\mathbf{a}}$ \\
\hline & & 60 & $6.77 \pm 0.20^{\mathrm{a}}$ & $12.87 \pm 0.47^{\mathbf{a}}$ & $71.80 \pm 0.42^{\mathbf{a b}}$ & $22.83 \pm 1.00^{\mathrm{a}}$ & $31.83 \pm 0.60^{\mathrm{a}}$ \\
\hline
\end{tabular}

Values are expressed as mean \pm SE.

The values with different superscript letters within the same column significantly differ at $p<0.05$.

Table 5: White blood cells and platelets count after after partial and complete splenectomy in young and adult dogs

\begin{tabular}{|c|c|c|c|c|c|c|}
\hline & & & WBCs & Lymph & Neutrophil & Platelets \\
\hline \multirow{8}{*}{$\begin{array}{l}\text { Young } \\
\text { dogs }\end{array}$} & \multirow{4}{*}{$\begin{array}{c}\text { Complete } \\
\text { splenoctomy }\end{array}$} & 0 day & $8.83 \pm 0.18 \mathrm{~d}$ & $2.90 \pm 0.12 \mathrm{~d}$ & $6.73 \pm 0.33 \mathrm{~d}$ & $210.33 \pm 4.98 \mathrm{c}$ \\
\hline & & 10 day & $13.87 \pm 0.46 b$ & $5.10 \pm 0.15 b$ & $11.47 \pm 0.50 b$ & $358.33 \pm 10.93 a$ \\
\hline & & 30 day & $17.83 \pm 0.15 \mathrm{a}$ & $6.73 \pm 0.23 a$ & $16.10 \pm 0.23 a$ & $300.00 \pm 5.77 \mathrm{c}$ \\
\hline & & 60 day & $11.17 \pm 0.15 \mathrm{c}$ & $4.10 \pm 0.06 c$ & $9.93 \pm 0.43 c$ & $230.00 \pm 8.66 \mathrm{~b}$ \\
\hline & \multirow{4}{*}{$\begin{array}{c}\text { Partial } \\
\text { splenoctomy }\end{array}$} & 0 day & $7.90 \pm 0.12 \mathrm{c}$ & $3.23 \pm 0.12 \mathrm{c}$ & $7.83 \pm 0.18 b$ & $235.67 \pm 8.09 b$ \\
\hline & & 10 day & $9.03 \pm 0.09 \mathrm{a}$ & $4.27 \pm 0.12 b$ & $9.40 \pm 0.23 \mathrm{a}$ & $316.67 \pm 12.02 \mathrm{a}$ \\
\hline & & 30 day & $8.63 \pm 0.12 b$ & $5.40 \pm 0.23 \mathrm{a}$ & $10.03 \pm 0.29 \mathrm{a}$ & $295.00 \pm 2.89 \mathrm{a}$ \\
\hline & & 60 day & $7.07 \pm 0.09 \mathrm{~d}$ & $2.97 \pm 0.07 \mathrm{c}$ & $7.47 \pm 0.23 b$ & $210.00 \pm 5.77 \mathrm{~b}$ \\
\hline \multirow{8}{*}{$\begin{array}{l}\text { Adult } \\
\text { dogs }\end{array}$} & \multirow{4}{*}{$\begin{array}{c}\text { Complete } \\
\text { splenoctomy }\end{array}$} & 0 day & $13.27 \pm 0.45 \mathrm{~d}$ & $3.67 \pm 0.22 \mathrm{~d}$ & $9.63 \pm 0.30 \mathrm{c}$ & $403.67 \pm 8.76 \mathrm{c}$ \\
\hline & & 10 day & $16.90 \pm 0.12 b$ & $4.77 \pm 0.13 c$ & $12.13 \pm 0.18 b$ & $576.67 \pm 14.53 b$ \\
\hline & & 30 day & $22.03 \pm 0.32 \mathrm{a}$ & $6.73 \pm 0.18 \mathrm{a}$ & $17.00 \pm 0.53 \mathrm{a}$ & $658.33 \pm 19.22 \mathrm{a}$ \\
\hline & & 60 day & $15.47 \pm 0.37 \mathrm{c}$ & $5.53 \pm 0.12 b$ & $12.27 \pm 0.58 b$ & $576.67 \pm 14.53 b$ \\
\hline & \multirow{4}{*}{$\begin{array}{c}\text { Partial } \\
\text { splenoctomy }\end{array}$} & 0 day & $12.60 \pm 0.32 b$ & $3.60 \pm 0.26 c$ & $9.30 \pm 0.32 \mathrm{c}$ & $221.67 \pm 9.28 \mathrm{c}$ \\
\hline & & 10 day & $16.27 \pm 0.46 a$ & $5.60 \pm 0.15 a$ & $11.47 \pm 0.18 \mathrm{a}$ & $332.33 \pm 15.84 b$ \\
\hline & & 30 day & $15.50 \pm 0.26 \mathrm{a}$ & $4.93 \pm 0.26 \mathrm{ab}$ & $10.43 \pm 0.18 b$ & $429.67 \pm 15.50 \mathrm{a}$ \\
\hline & & 60 day & $13.33 \pm 0.24 b$ & $4.13 \pm 0.34 b c$ & $9.77 \pm 0.43 b c$ & $338.33 \pm 19.22 b$ \\
\hline
\end{tabular}



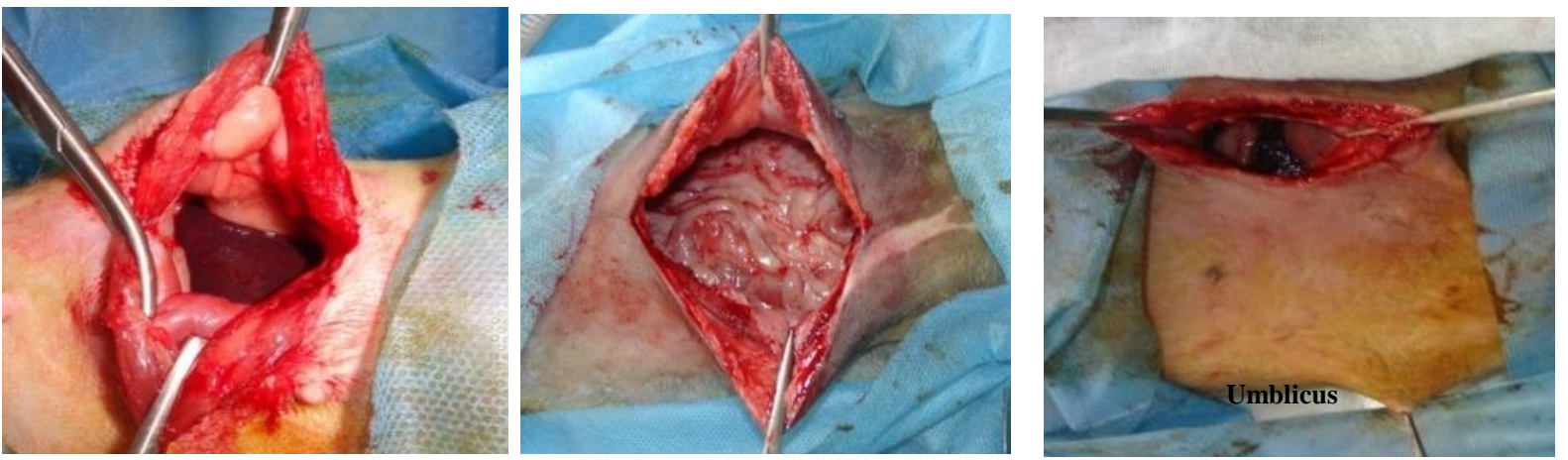

Fig.1: Abdominal incision for located spleen:

Fig.1-a: Midline abdominal Fig.1-b: Midline abdominal incision in young dog located incision in adult dog spleen was not spleen

located for its lateral position

Fig.1-c: Left sub-costal abdominal incision in adult dog located spleen
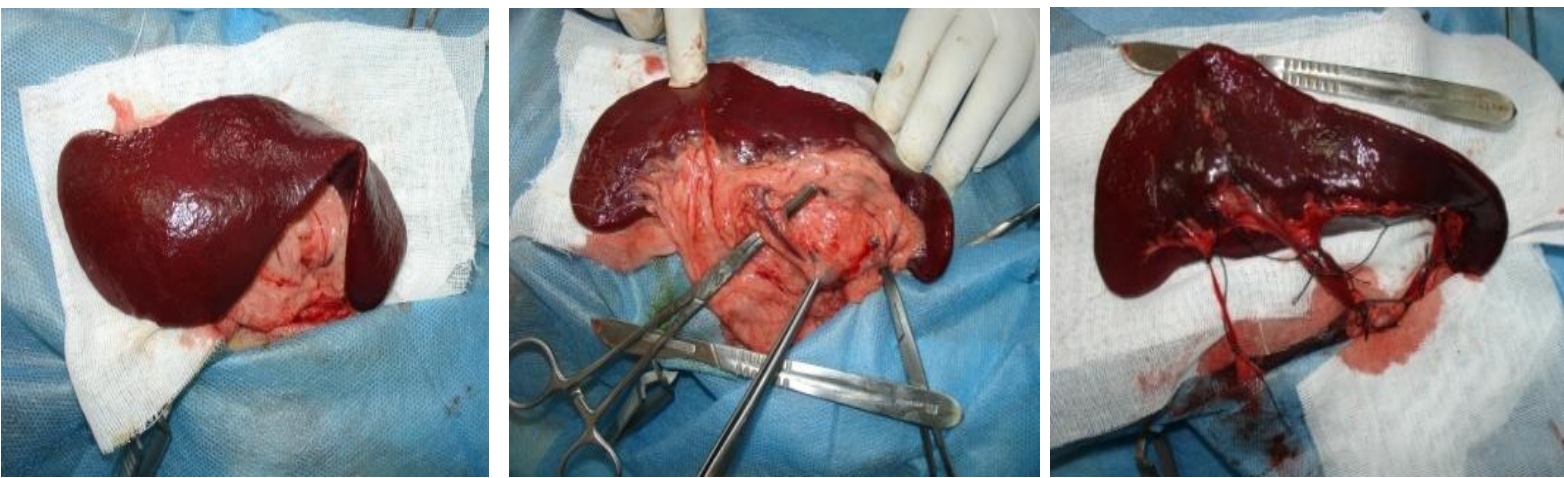

Fig.2: Isolation of the spleen extra abdominal for complete splenectomy:

Fig.2-a: The abdominal incision Fig.2-b: Identify and isolation of was covered with moisted the splenic artery and vein and laparatomy sponges after isolate the short gastric vessles spleen
Fig.2-c: Individual ligation to vessels $1-2 \mathrm{~cm}$ from their entrance into splenic parenchyma
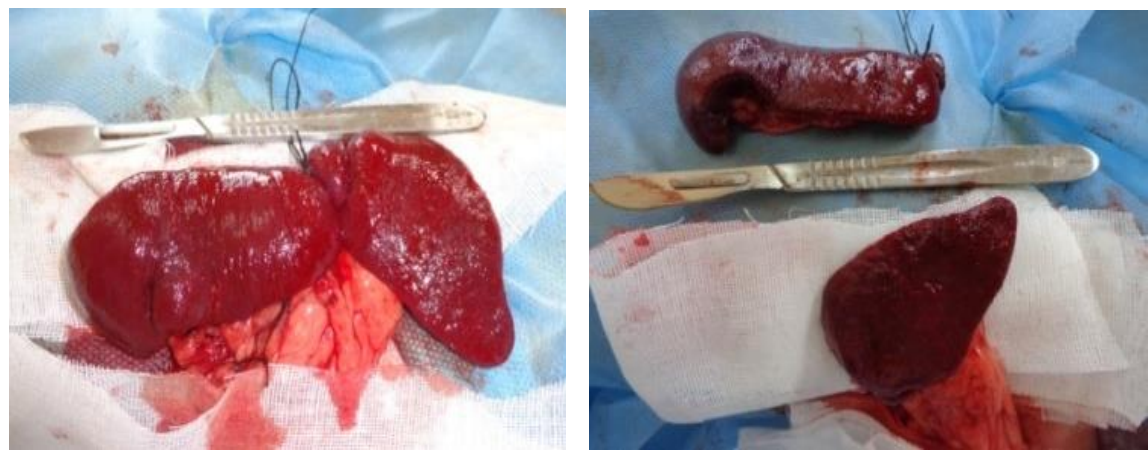

Fig.3: partial splenectomy

Fig.3-a: Double ligation to splenic Fig.3-b: The spleen was incised parenchyma after detecting to resected between two ligation to ensures part and closure to its vesseles complete hemostasis 


\section{DISCUSSION}

The effect of splenectomy either partial or complete on some biochemical parameters was studied. The reduction of serum albumin post operation (data presented in table 1) was as part of the systemic response to surgical trauma which was agreed with previous studies. There was a reduction of albumin in the postoperative peripheral blood relative to its values in the splenic vein and preoperative peripheral blood in schistosomal patients undergoing splenectomy (Petroianu et al., 2004). Concerning iron balance, serum iron concentration was decreased with significant increase in serum ferritin, transferring, and ceruloplasmin in dogs under both types of splenectomy (table 2). These results were in accordance with Kolnagou et al. (2013) who found that total body iron storage capacity is reduced, whereas serum ferritin and iron concentration in other organs appears to be increased in thalassemia major patients following splenectomy. Serum ferritin was increased when iron level was low; this exploration was parallel to that of De Domenico et al. (2011) who showed that ferritin secretion results when cellular ferritin synthesis occurs in the relative absence of free cytosolic iron. A compensatory increase in the serum level of transferring and ceruloplasmin is due to increased hepatic synthesis of both proteins following decreased serum iron level.

Concerning hepatic functions, splenectomy can regenerates the hepatic functions assessed by low enzymatic activity (ALT, AST) and bilirubin level (Table 3). These results came in agreement with Elsebae and Abu-Zekri, (2008) who stated that the activities of ALT, AST, and ALP and level of albumin were significantly decreased while serum bilirubin increased following splenectomy in patients with chronic hepatitis $\mathrm{C}$ viral infections. The present study showed significant decrease in RBCs and $\mathrm{Hb}$ in completely splenectomized dogs in contrary partially dogs these results coincides with results observed by Chaudhry et al. (1997). Complete splenectomy cause decrease in red blood cell values, even below physiological values (Jain, 1993). MCV and MCHC significantly increase after operation, these results agreed with results obtained by Knežević et al. (2002). The aging of erythrocyte lead to change in its plasma membrane causing retention of fluids inside the cell, so aged RBCs have higher mean corpuscular volume values. Total splenectomy lead to increased number of circulating old red blood cells, this combined with sever reticulocytosis, led to high mean corpuscular volume values in pigs (PoljičakMilas et al., 2012). Platelets count showed significant increase in completely splenectomized small and large dogs soon after operation, these results also obtained by Karagülle et al. (2007). As about one third of total platelets are sequestered in spleen, also spleen is the site of platelet destruction, so after removal of splenectomy, thrombocytosis will develop. Ichikawa et al. (1998) indicated that, gradual increase in thrombopoietin, IL-1 and erythropoietin levels in splenectomized patients followed by increase in platelets number. It has been stated that splenectomy causes changes in the number of white blood cells with later leukocytosis The Peripheral WBC counts in animals following partial splenectomy were significantly different from those found in animals subjected to total splenectomy at the same time periods (Bessler et al., 2004). There are several mechanisms participate in the excess of WBCs after splenectomy, decrease in apoptotic cell death rate following splenectomy (Djaldetti et al., 2003). Additional mechanism is cell migration; small lymphocytes migrate from peripheral blood into lymphoid tissues and into the spleen and are released back to the blood (Rannie and Donald, 1977). We concluded that partial splenectomy is preferred over total splenectomy when possible because it preserves splenic function. The spleen normally contains a reservoir of red blood cells (RBCs), has hematopoietic capabilities, has important phagocytic functions, and is helpful in maintaining immunocompetence; total splenectomy eliminates these beneficial actions.

\section{REFERENCES}

Bessler, H.; Bergman, M.; Salman, H.; Beilin, B. and Djaldetti, $M$. (2004): The relationship between partial splenectomy and peripheral leukocyte count. J Surg Res, 122, 49-53.

Chaudhry, N.I.; Khan, M.A.; Kausar, S. and Iqbal, K. (1997): Experimental study on hemogram and chemistry profile in splenectomized dogs. Pak Vet J, 1, 17 -23.

De Domenico, I.; Vaughn, M.B.; Paradkar, P.N.; Lo, E.; Ward, D.M. and Kaplan, J. (2011): Decoupling ferritin synthesis from free cytosolic iron results in ferritin secretion. Cell Metab, 13, 57-67.

Djaldetti, M.; Bergman, M.; Salman, H.; Cohen, A.M.; Fibach, E. and Bessler, H. (2003): On the mechanism of post-splenectomy leukocytosis in mice. Eur J Clin Invest, 33, 811-817.

Elsebae, M.M. and Abu-Zekri, N.B. (2008): A study of the effect of splenectomy on hepatic functional reserve and structural damage in patients with chronic hepatitis $\mathrm{C}$ virus infection by non-invasive serum markers. A prospective study. Int J Surg, 6 (5), 362-366.

Finch, C.A.; Bellotti, V. and Stray, S. (1986): Plasma ferritin determination as a diagnostic tool. West J Med, 145, 657-663.

Harari, J. (1999): Baço, p.244-248. In: Harari J. (Ed.), Cirurgia de Pequenos Animais. Artes Médicas Sul, Porto Alegre. 
Ichikawa, N.; Kitano, K.; Shimodaira, S.; Ishida, F.; Ito, T. and Kajikawa, S. (1998): Changes in serum thrombopoietin levels after splenectomy. Acta Haematol, 100, 137- 41.

Jain, N.C. (1993): Essentials of Veterinary Hematology. Williams and Wilkins, ISBN 081211437, USA.

Kaplan, A. and Savory, J. (1965): "Evaluation of a cellulose-acetate electrophoresis system for serum protein fractionation". Clin Chem, 11 (10), 937-942.

Karagülle, E.; Hoşcoşkun, Z.; Kutlu, A.K.; Kaya, M. and Baydar, S. (2007): The effectiveness of splenic autotransplantation: an external study. Turk J Traum Emerg Surg, 13, 13-19.

Khan, P.N.; Nair, R.J.; Olivares, J.; Tingle; L.E. and Li, Z. (2009): Postsplenectomy reactive thombocytosis. Baylor University Medical Center Proceedings, 22, 9-12.

Knežević, S.; Stefanović, D.; Petrović, M.; Djordjević, Z.; Matić, S.; Artiko, V.; Milovanović, A. and Popović, M. (2002): Autotransplantation of the spleen. Acta Chirurgica Jugoslavica, 49, 101-106.

Kolnagou, A.1.; Michaelides, Y.; Kontoghiorghe, C.N. and Kontoghiorghes, G.J. (2013): The importance of spleen, spleen iron, and splenectomy for determining total body iron load, ferrikinetics, and iron toxicity in thalassemia major patients. Toxicol Mech Methods, 23 (1), 34-41.

Lizana, J. $\quad$ and Hellsing, $K$. (1974): Manual immunoephelometric assay of proteins, with use of polymer enhancement. Clin Chem, 20 (9), 1181-1186.

Petroianu, A. (2003): Tratamento cirorgico da hipertensº porta na esquistossomose mansoni. Rev Soc Bras Med Trop, 36 (2), 253-265.

Petroianu, A.; Alberti, L.R.; Zac, R.I. and Andrade J'nior, J.C.C.G. (2004): Influência do trauma cirrgico na concentraço sérica de albumina no psoperatrio imediato. Rev Col Bras Cir, 31 (3), 194-9.

Poljičak-Milas, N.; Vujnović, A.; Migić, J.; Vnuk, D. and Kardum, M. (2012): The Effects of Splenectomy and Autologous Spleen Transplantation on Complete Blood Count and Cell Morphology in a Porcine Model. Hematol Sci Pract, Published in print edition March.

Rannie, G.H. and Donald, K.J. (1977): Estimation of the migration of thoracic duct lymphocytes to non-lymphoid tissues. Cell Tissue Kinet, 10, 523-541.

Sipka, J.S.; Brath, E.; Toth, F.F.; Aleksza, M.; Kulcsar, A. and Fabian, A. (2006): Cellular and serological changes in the peripheral blood of splenectomized and spleen autotransplanted mice. Transpl Immuno, 16 (2), 99-104.

Tillson, D.M. (2003): Spleen. In: Textbook of Small Animal Surgery, Slatter, D.H, Ed., pp. (10461062), Saunders Co. LTd, ISBN 9780721686073, Philadelphia.

Wolf, P.L. (1982): Ceruloplasmin: methods and clinical use. Crit Rev Clin Lab Sci, 17 (3), 229-45.

\section{التأثيرات الكيميائية الحيوية والدموية لاستئصال الطحال الجزئى والكلى فى الكلاب

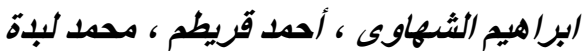

E-mail: biochemistry232@yahoo.com Assiut University web-site: www.aun.edu.eg

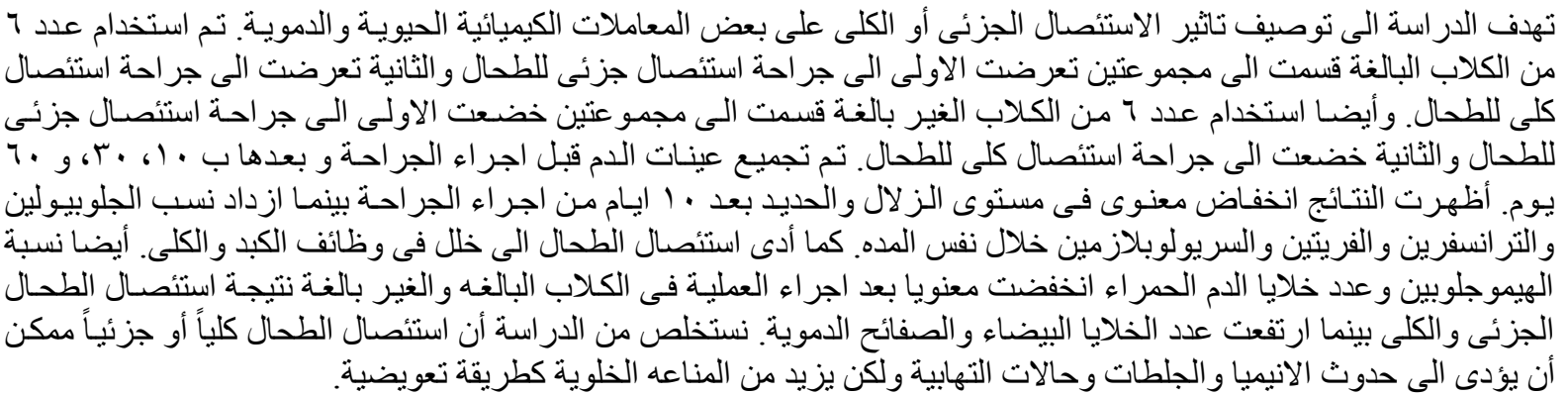

